\title{
Observatorio
}

\section{Elegía del centro de documentación de prensa}

\author{
Por Nora Paul
}

\begin{abstract}
Resumen: Con la biografía de la documentalista Liz Donovan, fallecida en 2008, se hace un recorrido por la profesión de documentalista de prensa, y se describe la evolución de las redacciones de los periódicos a lo largo de 40 años. Han cambiado los sistemas y los recursos de información-desde el soporte papel hasta la llegada de internet y la Web 2.0-, sin embargo también ha cambiado la situación económica de la prensa, que tiene que hacer restricciones. Desgraciadamente, con la idea de que "en internet está todo", las empresas valoran poco los servicios de documentación y los cierran o los diezman, perdiendo así un gran capital de saber hacer y de calidad.
\end{abstract}

Palabras clave: Centro de documentación, Profesionales, Valoración profesional, Prensa, Periódicos, Diarios, Medios de difusión, Evolución histórica, Recesión económica, Crisis de la prensa escrita.

\section{Title: Elegy for the news library}

Abstract: The biography of news researcher Liz Donovan, who died in 2008, serves as a thread of a journey through the profession of news librarian and describes the evolution of newspaper newsrooms throughout 40 years. Information systems and resources have changed -from paper clippings to the Internet and Web 2.0-, but the economic situation of the press has also changed, leading to cutbacks. Unfortunately, with the mistaken idea that "everything is on the Internet", news companies are devaluing the contributions made by information professionals to newspaper content, and often close or decimate the news libraries, thus losing an important amount of know-how and quality.

Keywords: Information centre, News library, Profession, Professional value, Professional profile, Newspapers, Media, History, Economic recession, Crisis of the press.

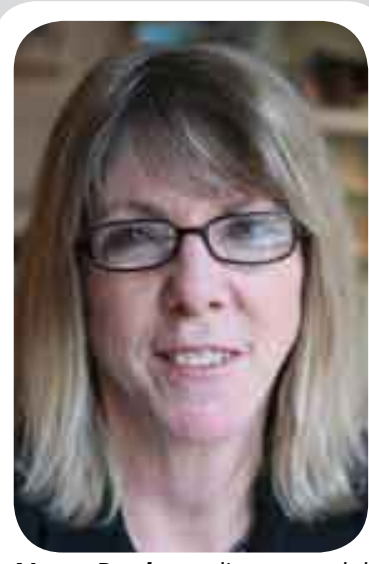

Nora Paul es directora del Institute for New Media Studies, de la University of Minnesota. Anteriormente fue documentalista de prensa en el diario Miami Herald. Es autora de numerosos artículos sobre documentación en medios de comunicación y sobre la profesión de documentalista de prensa.

Paul, Nora. "Elegía del centro de documentación de prensa". El profesional de la información, 2009, mayo-junio, v. 18, n. 3, pp. 249-253.

DOI: 10.3145/epi.2009.may.01

LA DOCUMENTALISTA DE PRENSA Liz Donovan murió a principios de diciembre de 2008, a los 64 años de edad. En los cuarenta años que trabajó en las redacciones de periódicos vio y vivió los cambios que la tecnología trajo en la práctica de los servicios de documentación.

Liz comenzó su carrera como documentalista del diario Washington post a finales de 1960. Era una época de búsquedas en papel, recortes de prensa guardados en carpetas y sobres en cajones y más cajones de archivadores en servicios de documentación centralizados.

Liz fue la persona de Bob Woodward y de Carl Bernstein cuando necesitaron seguir la evolución de los acontecimientos en el caso Watergate, cuya cobertura condujo a la caída final del presidente Richard Nixon.

He aquí cómo Liz describió su trabajo:
"Casi todos los días pasaba un par de horas recortando y archivando las menciones al asunto Watergate de todas las principales revistas y diarios a los que teníamos acceso, y trabajé con Carl y Bob en el seguimiento de los eventos. Cuando el caso llegó a la situación de crisis y Carl y Bob publicaron su libro en la primavera de 1974, los recortes llenaban ya un armario entero"

En la década de 1980 se fue a Miami y fue contratada por el diario Miami Herald como documentalista, donde ayudó en la transición de los recortes de papel

"Cuando explotó el escándalo Watergate mis recortes de prensa llenaban un armario entero" (Liz Donovan) 
de noticias al archivo electrónico. Esa época requería un modo de pensar transversal, analizando minuciosamente los sistemas de información existentes. Los documentalistas tenían que ser muy cuidadosos en el control de los recursos, tanto de los archivos de recortes como de las nuevas bases de datos electrónicas. Se necesitaba habilidad en las búsquedas online por texto libre, lo mismo que al utilizar palabras clave en los archivos de recortes, y también había que familiarizarse con el recién iniciado acceso remoto a bases de datos de noticias de hosts online como Dialog y Nexis. Esto permitió a los reporteros del periódico añadir más contexto a sus noticias, tratar no sólo lo que había sucedido en su propia ciudad, sino hacerse eco de cómo los asuntos se habían reflejado en otras ciudades.

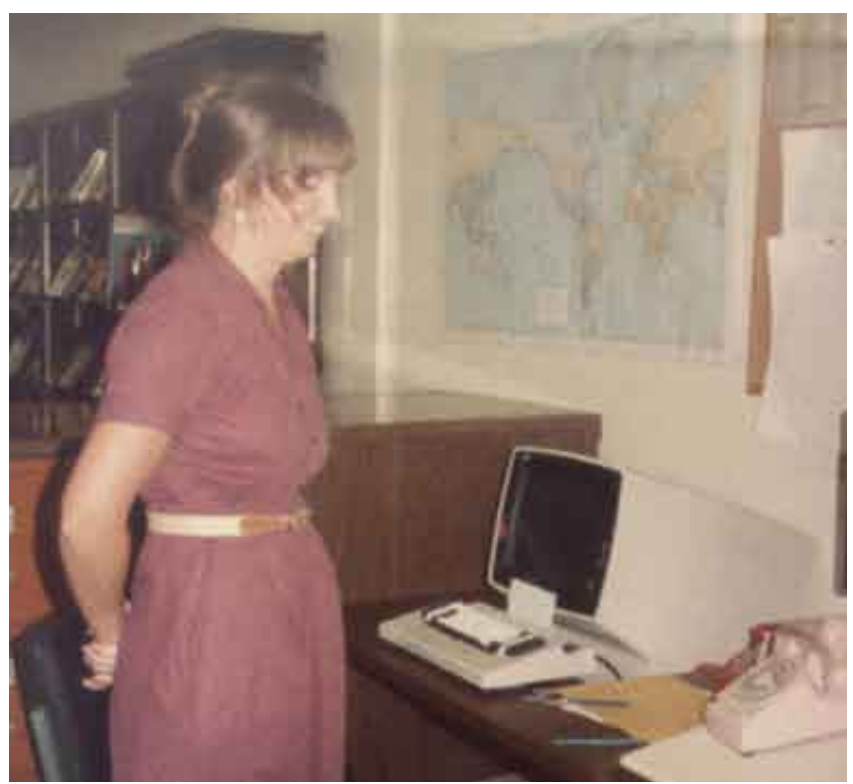

Liz Donovan frente a su primer ordenador en 1983

\section{Gatekeeper}

Al principio las bases de datos online eran difíciles y costosas, y el equipo necesario no estaba disponible para todos, sólo estaba en el centro de documentación. El documentalista era un intermediario esencial entre el reportero y todo el fondo de historias y documentos que necesitaban. Liz fue, una vez más, la persona a la que era obligado acudir para realizar una pesquisa o una investigación.

Al incremento de acceso a las bases de datos y otras fuentes de noticias y artículos de revistas, se sumó a finales de los 80 s el acceso online a los registros públicos. Florida es un estado donde las leyes del "Government in the sunshine" protegen y promueven el acceso a los registros administrativos. Basándose en sus habilidades de búsqueda de documentos en papel, Liz añadió la búsqueda de noticias online a su repertorio. El acceso o petición online de los documentos primarios facilitó la profundidad y la minuciosidad de la investigación de los periodistas. Dado que todavía no había ordenadores personales, el acceso a estos recursos se hacía a través del centro de documentación. El documentalista era un verdadero socio en el proceso del reportaje, suministrando una gran variedad de artículos de fondo y documentos originales provenientes de una amplia gama de fuentes.

El decenio de 1990 trajo internet a las masas. Los periodistas ya no trabajaron más en terminales "tontos" que sólo estaban conectados al sistema de producción. Empezaron a trabajar en ordenadores personales que les conectaban a un universo de información en rápido crecimiento. Liz encontró una nueva responsabilidad: orientar a los reporteros y editores sobre las mejores webs.

Mientras el acceso a las bases de datos online estaba limitado a unos pocos ordenadores en el diario al tratarse de servicios de búsqueda muy caros, Liz fue una information gatekeeper (guardián o responsable de información), un socio esencial para los reporteros que necesitaban localizar información. Pero en este mundo nuevo de bajo coste de acceso a la World Wide Web y su gran riqueza de información, hizo la transición de ser gatekeeper entre el periodista y la información que este necesita, a ser facilitadora de información, ayudando a los periodistas a desenvolverse por sí mismos.

Estaba ocurriendo un cambio de rol: de "camarera de información" -situación en la que los periodistas piden al documentalista la información que necesitan- a "guía de información". Los reporteros se encontraron con el acceso a través de sus ordenadores a archivos, bases de datos, estudios de centros de investigación y recursos de referencia inimaginables en el pasado.

Liz se adaptó a este cambio. Entendió que parte del rol de guía era encontrar, evaluar y organizar los recursos web, para lo cual utilizó su capacidad para evaluar críticamente y organizar los enlaces a los recursos más útiles. También ayudó a crear la intranet, que ahora es una parte importante de la estructura de la redacción. Fue lo que se llama una "information curator", seleccionando las mejores piezas para añadir a la colección.

\section{"Estaba ocurriendo un cambio de rol: de camarera de información, pasó a ser guía de información"}

\section{Formación de usuarios}

Otra faceta de su creciente papel como guía de información fue la de proporcionar formación. Liz tra- 


\section{Infomaniac Behind the News

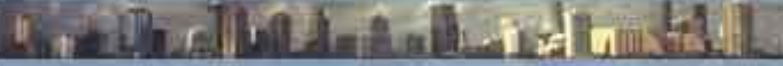

\section{Google}

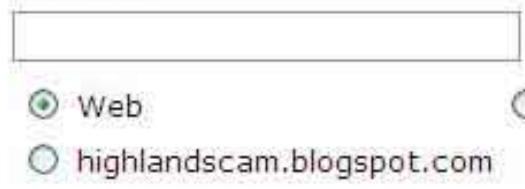

\begin{tabular}{|c|c|}
\hline Read "Obama Today" Blog & ePals Election Central \\
\hline $\begin{array}{l}\text { Follow the latest deyelopments in Barad } \\
\text { Obama's administration. }\end{array}$ & $\begin{array}{l}\mathrm{K}-12 \text { interactive and coll aborative activities for } \\
\text { the } 2008 \text { election }\end{array}$ \\
\hline$<>$ & Ads by $\mathrm{Goc}$ \\
\hline
\end{tabular}

H Saturday, November 29, 2008

\section{Holiday weekend diversion}

For something totally silly, especially if you're a fan of sites like Stuff White People Like, here's a new one: Stuff Joumalists Like. Like 'Professional Organizations':

Journalists need to connect with others who enjoy long talks about deadlines, copies and incompetent editors. That is why journalists like professional onganizations.

Groups like Society of Professional Journalists and Investigative Reporters and Editors give journalists a sense of community and a place to feel better about the career choices they have made.

(Via Miranda Writes.)
About

About the nevs, the Nel, blogs and journalism, new media, news research and other things I like, from a veteran news researcher with stops in DC, Mlami and nirw the Southem Highlands of Appdiachia.

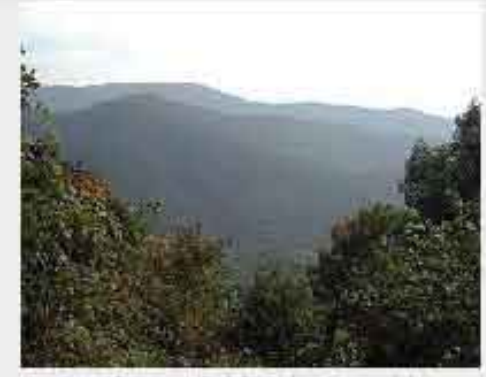

Southern Hiqhlands Cam bloq

About Me

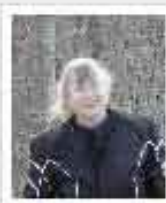

Name: liz donovan Location: Cherokee County, North Carolina, Unifed States View my complete profile

Labels: joumalism

Último post de Liz Donovan en su blog

bajó con los reporteros y editores para desarrollar sus habilidades de búsqueda online, siguiendo la máxima de "si das un pescado a un hombre comerá una vez, pero si le enseñas a pescar comerá siempre”. Orientó a los periodistas usando un lenguaje no técnico, adaptado a ellos, ayudándoles a dominar las tareas de búsqueda de la información que necesitan diariamente.

Trabajó con los periodistas en la creación de bases de datos que podrían ser usadas para proyectos de investigación más profunda. En 1999 formó parte del equipo de investigación sobre el fraude de votantes en el condado de Dade, que requería la construcción y el mantenimiento de una gran base de datos. Como resultado de sus contribuciones fue citada como integrante del equipo que ganó un premio Pulitzer (por segunda vez en su carrera) -el primero fue por la cobertura del Watergate-. Sus conocimientos sobre arquitectura de bases de datos fueron muy valorados.

Como los servicios que prestaba se fueron integrando más y más en el flujo de trabajo de los periodistas, decidió cambiar su despacho del servicio de documentación a la sala de redacción. Fue como un socio en la labor de búsqueda de información precisa, pertinente e integral para informar sobre noticias importantes.

\section{Bloguear}

Liz continuó usando las nuevas tecnologías. Comenzó su primer sitio web, al estilo de un blog, en 2000. Blogueó para el Herald en miamiherald.com y comenzó el blog "Infomaniac" -uno de los primeros 
sitios centrados en temas de investigación de noticias. Sus posts ampliaron su influencia desde la redacción del Miami Herald a un público mundial. Continuó incluso después de su jubilación del Herald en 2004 y hasta una semana antes de su muerte.

\section{http://newsresearch.blogspot.com/}

Si Liz no se hubiera jubilado, estoy segura de que se hubiera puesto a ayudar en la mejora de la web del Herald optimizando el motor de búsqueda -sus conocimientos de metadatos y palabras clave hubieran sido muy valiosos-. Estoy segura de que se habría entusiasmado aprovechando la expansión de las redes sociales tanto para la investigación como para crear nuevas formas de llegar al público. Liz se habría volcado completamente en Twitter.

Con la próxima ola de recursos internet y la promesa de la Web semántica de traer un mejor nivel de búsqueda contextual, sé que Liz habría estado justo en el centro, ayudando a la redacción a hacer el mejor uso posible de cada nuevo invento. Adoraba su trabajo. Fue la quintaesencia del documentalista de medios: detective, guía, gestora de información, productora de bases de datos, formadora, blogger... En las redacciones de todo el mundo hay otros como ella. Profesionales de la información que ayudan a sus compañeros periodistas a navegar por el siempre cambiante y en continua expansión mar de recursos de información, cuidando de los datos, formando y realizando búsquedas creativas.

\section{"Fue la quintaesencia del documentalista de medios: detective, guía, gestora de información, creadora de bases de datos, formadora, blogger..."}

\section{Declive de una profesión}

Bueno, por lo menos así era la cosa hasta hace más o menos un año. Me temo que si Liz no se hubiera retirado, aun con todas sus habilidades y un gran historial de valiosa contribución a la redacción, se hubiera sumado a las filas de documentalistas despedidos o prejubilados. Cuando las redacciones de los medios empiezan a tener que hacer equilibrios para contrarrestar la hemorragia de sus presupuestos de funcionamiento, el centro de documentación a menudo es de los primeros en sufrir las consecuencias.

Triste es decirlo pero la muerte de Liz, por desgracia, es emblemática de la situación actual de los centros de documentación de las redacciones en los EUA, muchos también han muerto o están agonizantes.
The Wall Street journal cerró su unidad de documentación. La del Philadelphia pasó de una plantilla de 15 a 2. Desde hace tiempo los jefes de los centros de documentación están haciendo ofertas a los empleados para que se vayan, tirando de esta manera años de conocimientos y de sentido de la noticia, para continuar trabajando con un personal diezmado. En las redacciones hay un vacío de visión y de gestión de la información.

Tim Rozgonyi, director de documentación del St. Petersburg times, envió un mensaje a la lista News Library (newslib) después de haber oído decir a Leslie Norman, la documentalista del Wall Street journal, que su servicio de documentación se iba a cerrar. Creo que hace el elocuente trabajo de explicar el papel que un documentalista de prensa desempeña en una organización periodística, y por qué la reducción en el mejor caso, o la eliminación en el peor, de este papel fundamental tendrá repercusiones negativas para las redacciones que intentan crear un nuevo futuro. He aquí unos extractos del anuncio hecho por Leslie Norman de la clausura:

\section{De: Leslie Norman}

5 de febrero de 2009

Lamento informar que el servicio de documentación del WSJ dejará de existir el 23 de marzo de 2009.

Hoy nos dieron el aviso de nuestro despido. Yo he pedido continuar como consultora sólo con el sueldo, sin los beneficios. La decisión está en manos de la dirección...

Cuando pregunté quién va a hacer las búsquedas para los periodistas, se me dijo, "nadie”. Los periodistas probablemente utilizarán un producto de LexisNexis llamado Due Diligence Dashboard (recuerda lo que tu mamá te dijo: "si no puedes decir algo agradable..., no digas nada...”). Pero ese servicio no puede sustituir nuestros conocimientos de cómo buscar, con todos los trucos que hemos aprendido con los años. Nos imaginamos que los periodistas tratando de hacer sus propias búsquedas probablemente gasten 10 veces el dinero que la empresa se ahorra sin nosotros.

Me gusta mucho mi trabajo pero no creo que vaya a encontrar un empleo en un centro de documentación de prensa en un futuro próximo. Cada día es diferente y un reto. No quiero ofender, pero trabajando en una biblioteca pública me volvería loca.

Estoy también preocupada por los microfilms. Desde que termina la base de datos histórica de ProQuest hasta que comienzan nuestros archivos en pdf hay cerca de 10 años en los que la única forma física de los documentos es en microfilm.

Hay tantas pequeñas cosas acerca de lo que hacemos... ¿cómo explicarlas o cómo escribir sobre ellas? 
Y eso es lo que Tim respondió:

Siento escuchar esta noticia. Te deseo a ti y a tu familia todo lo mejor.

Tu nota, triste, también representa lo que es la documentación de medios. A pesar de estar comprensiblemente preocupada por lo que pueda depararte el futuro, estás imaginando cómo esto afectará a la redacción del periódico, ¿qué pasará con el microfilm del WSJ y cómo en las próximas semanas se podrían cuantificar $y$ documentar los conocimientos especializados únicos que tú tienes, a fin de que no sufra la organización que ha considerado que tú no eres esencial?

La verdad es que no puedes. Los documentalistas de prensa tenemos una perspectiva totalmente distinta de la de las redacciones en las que trabajamos. Nosotros realizamos un servicio, que no se considera necesariamente un valor básico para procesar las noticias. Sabemos enfocar nuestra atención hacia el pasado y hacia el futuro, y pensamos en términos de décadas, mientras que la mayoría del personal nuevo sólo piensa en hoy y en mañana, siendo el ayer sólo un recuerdo lejano.

Esa es la bendición y la maldición del documentalista de prensa: tenemos una sensibilidad y un conjunto de valores que generalmente no son compartidos por la comunidad a la que servimos.

\section{"El documentalista de prensa tiene una sensibilidad y unos valores que generalmente no son compartidos por la comunidad a la que sirve"}

Las personas que toman la decisión de cerrar los servicios de documentación o disminuir su dotación de personal se mueven en un mundo diferente al que habitamos los documentalistas. Problemas que se han tardado décadas en resolver, ellos creen que son soluciones a corto plazo para el siguiente trimestre, sin entender el daño a largo plazo que pueden hacer deshaciéndose de las personas que ellos ven sólo como "chequeadores de hechos" o administrativos bien pagados que introducen datos. Están demostrando la misma miopía que ha causado que los periódicos sean menos relevantes de lo que eran en el pasado y que estén en riesgo de perder toda posibilidad de conectar con la generación de jóvenes.
¿Quién va a llenar el agujero que dejas tras de sí, Leslie? Se necesitaría a alguien que valore el compartir información profusamente y de manera proactiva, alguien que realmente entienda que el contenido de las noticias es la única cosa de valor duradero que las organizaciones de medios dejan tras de sí, alguien que entienda el valor inestimable del contenido de la noticia de hoy y esté dispuesto a tomar todas las medidas necesarias para asegurarse de que se conserva para beneficio de la organización y para la posteridad.

¿Hay alguna persona así en las actuales redacciones? Bueno, sí hay, y se llaman documentalistas. $Y$ el hecho de que nuestros números estén disminuyendo es un triste testimonio del estado de los periódicos de hoy.

Una vez más, te deseo todo lo mejor, Leslie. Tú eres claramente una de esas personas que encarnan las impresionantes cualidades que hacen de los documentalistas de prensa ese singular e importante ingrediente en la receta para el éxito de las organizaciones que procesan noticias.

\section{Cúidate}

Tim

Leslie fue la $\mathbf{L i z}$ Donovan del Wall Street Journal. Cada Redacción necesita una Leslie, o una Liz, o un Tim, Sharon, Kathy, Alan, Richard... En un entorno en el que navegar por la información es más complicado que nunca, las empresas de información y de noticias devalúan las contribuciones de los profesionales de la información.

Liz se sentiría más decepcionada.

Nora Paul, Institute for New Media Studies, School of Journalism and Mass Communication, University of Minnesota.

206 Church St. SE, 313 Murphy Hall

Minneapolis, MN 55455

Tel.: + 1-6126248593

http://www.inms.umn.edu npaul@umn.edu 


\section{doc6 en 30 segundos}

\section{Nuestra misión}

Contribuir a aumentar la eficiencia de empresas y organizaciones a través de una eficaz gestión de la información y el conocimiento.

\section{La empresa}

Creada en el año 1988, especializada en gestión de la información, la documentación y el conocimiento.

Certificada con la ISO 9001:2000 por Bureau Veritas desde

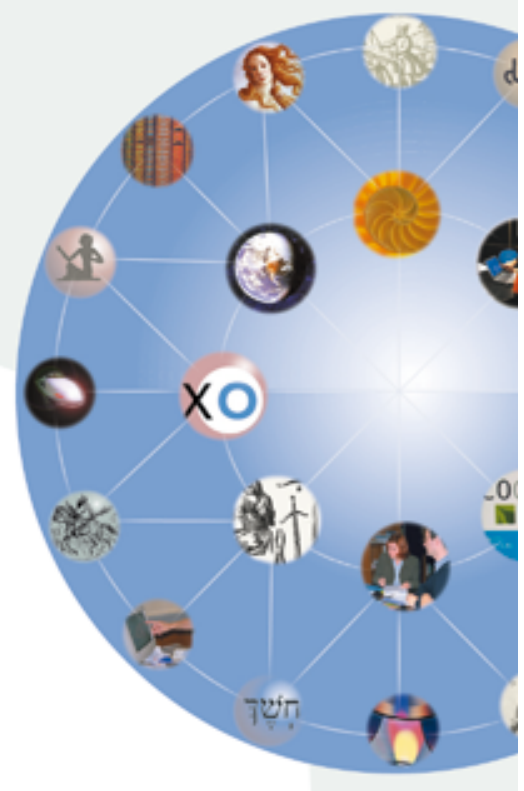

Septiembre del 2000 en las siguientes actividades:

a CONSULTORES EN RECURSOS DE INFORMACIÓN

a GESTIÓN DEL CONOCIMIENTO

Q INTRANET/EXTRANET/INTERNET

a EDICIÓN ELECTRÓNICA

- EXTERNALIZACIÓN DE SERVICIOS DOCUMENTALES

a PROVEEDORES DE RECURSOS ELECTRÓNICOS DE INFORMACIÓN

- SOLUCIONES EN GESTIÓN DOCUMENTAL

a CURSOS DE FORMACIÓN

\section{Nuestros valores}

Innovación permanente.

Satisfacción del cliente. Calidad

Profesionalidad / Cooperación con el cliente

Confidencialidad

Responsabilidad social y valores éticos

\section{Los clientes}

El principal activo de la empresa, junto con sus empleados.

Más de 900 clientes: empresas, laboratorios, administraciones públicas, universidades, hospitales, bufetes de abogados, museos, fundaciones, colegios profesionales.

\section{Nuestros profesionales}

Un equipo de más de 120 profesionales.

Una eficaz suma de conocimientos: informáticos y documentalistas con experiencia.

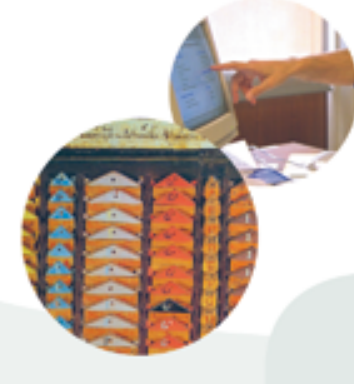

El $75 \%$ son licenciados superiores o diplomados

en informática o documentación.

\section{\begin{tabular}{llll}
1 & 9 & 8 & 8 \\
3 & 10 & 0 & 8 \\
\hline
\end{tabular}}

DOC5 SA. ha recibido la etiqueta EUREKA de $1+D$ para el proyecto COGNOS (n. 2734) de metodologia para el desarrollo de mapas del conocimiento en las organizaciones. 